Principales dates

19 octobre 1862 - Naissance d'Auguste à Besançon

5 octobre 1864 - Naissance de Louis à Besançon

1881

1894

1895

1903

Commercialisation des Plaques Étiquettes-Bleues

Invention du cinématographe

Premier film des frères Lumière

Invention de l'autochrome

6 juin 1948 - Décès de Louis à Bandol

10 avril 1954 - Décès d'Auguste à Lyon

\title{
Louis ef Auguste, les frères Lumière
}

Riad Haidar, haidar@onera.fr

Ingénieurs français, chercheurs et inventeurs prolifiques, industriels avisés, les frères Lumière ont joué un rôle de premier plan dans l'histoire du cinéma et de la photographie.

I l existe des coïncidences qui feraient croire au destin. Qu'on en juge : autour de la place située dans la boucle de Besançon, à quelques dizaines de mètres les unes des autres, on trouve les maisons natales du poète Charles Nodier, du député Charles Fournier et également de Victor Hugo... C'est également sur cette place, alors modeste et qui ne s'appelait pas encore Victor-Hugo mais Rondot Saint-Quentin (car il est dit que l'on dansait en rond au pied de la chapelle Saint-Quentin), qu'Auguste et Louis voient le jour, respectivement le 19 octobre 1862 et le 5 octobre 1864 , chez les Lumière, jeune famille parisienne qui vient de s'installer en Franche-Comté. Ils sont les premiers enfants de Jeanne Joséphine Costille, et d'Antoine Lumière, anciennement peintre d'enseignes et qui s'est récemment établi comme photographe. En 1870, la famille Lumière fuit I'Est de la France devant la menace prussienne, et s'installe à Lyon: Antoine ouvre un studio rue de la Barre, sur un terrain lové aux Hospices civils.

Ce père artisan et un peu artiste, gros travailleur, charismatique et bon vivant, joue un rôle décisif dans l'éveil intellectuel des deux frères, et s'impose comme un modèle de réussite. En effet, si ses débuts à Lyon sont difficiles, la grande qualité de ses portraits, qu'il obtient grâce à la lumière artificielle installée dans son studio, établit rapidement sa notoriété et lui procure une réelle aisance matérielle.

Auguste et Louis aident leur père dans son travail, et acquièrent une formation de techniciens à l'École municipale des Sciences et des Arts Industriels, dite la Martinière, installée sur le site de I'ancien couvent des Augustins de Lyon. Ils y développent leur goût pour la technique et la recherche. Auguste en sort en 1878, diplômé en physique ; et Louis, deux ans plus tard, en chimie. Sous la férule de leur père, les deux frères mettent au point une formule de plaques photographiques sèches à base de gélatino-bromure d'argent, afin de remplacer le collodion humide de Le Gray et Archer, alors populaire mais qui est d'une utilisation très contraignante (entre la préparation et le développement, l'opérateur ne dispose que d'une vingtaine de minutes au plus). Le renfort de ses fils donne ainsi des ailes à Antoine qui, en entrepreneur audacieux, décide de passer au stade industriel. En 1881, il quitte le studio de la rue de la Barre pour s'installer dans une ancienne chapellerie à Monplaisir, qu'il transforme en usine de plaques photographiques. Malgré des débuts difficiles, avec une production assurée au début uniquement par les membres de la famille (Antoine et Auguste dans le studio du centre-ville, Louis, sa mère et ses sœurs s'occupant des émulsions à l'usine, tous travaillant 18 heures par jour), la commercialisation des fameuses Plaques Étiquettes-Bleves Lumière est un immense succès, qui fonde la fortune familiale. Véritable consécration, I'entreprise Lumière et fils participe à l'exposition universelle de Paris en 1889, celle pour laquelle Eiffel construit sa tour. Louis et Auguste, ou plutôt les frères Lumière comme on commence à les appeler, sont des inventeurs prolifiques, et déposent de nombreux brevets, notamment dans le domaine de la photographie. Les liens filiaux sont forts, et ils ne quittent la maison familiale qu'en 1893, I'année de leur mariage avec les soeurs Winckler, Louis en février et Auguste en août. 


\section{Le cinéma}

En 1894, Antoine assiste à Paris à une démonstration du kinétoscope, I'appareil qui permet de voir, mais en spectateur solitaire, les films qu'Edison et son assistant Dickson ont enregistrés à l'aide de leur kinétogaphe. Il assiste également à une projection des premiers dessins animés du cinéma, que Reynaud présente dans son Théâtre Optique.

Véritablement impressionné, il perçoit très vite que cette image animée est un marché $d^{\prime}$ avenir pour peu que l'on parvienne à la projeter sur grand écran devant un public assemblé. Son enthousiasme est contagieux; il le transmet à ses fils. Louis met alors toute sa créativité à concevoir, durant l'été 1894 et en collaboration avec le chef mécanicien Charles Moisson, le mécanisme ingénieux qui réussit la provesse de matérialiser l'intuition paternelle, tout en se différenciant nettement du système breveté par Edison. D'ailleurs, s'ils présentent d'abord leur appareil sous le nom de « kinétographe Lumière » ou « kinétoscope Lumière », les frères Lumière s'émancipent très vite de la filiation d'Edison, et le baptisent « cinématographe ». Ils en déposent les droits le 13 février 1895, et entrent ainsi de plain-pied dans I'Histoire comme les inventeurs du cinéma en tant que spectacle photographique en mouvement projeté devant un public assemblé.

\section{LE Cinematographe}

\section{SALOK INDIIN cenes cans \\ 14. Bowbonat das Copucinon. "1 \\ PARIS}

Cet oppareil, invente par MM. Auguste et Louis Lumitre, permel de recueillir. par des series Stpreuoes instontantes, lous les mouoements qui, pendant un temps donnt, se sont suectdt dewant Pobjeclif, el de reproduire ensuile ces mouvements en projetant, grandear nalurelie, devant une salle entitre, leurs images sur un teran.

Leur premier film est La Sortie des Usines Lumière, tourné par Louis le 19 mars 1895 à la rue Saint-Victor (aujourd'hui rue du PremierFilm, à l'emplacement de l'actuelle Cinémathèque de Lyon). Le film est montré pour la première fois, en privé, le 22 mars 1895 dans les locaux de la Société d'Encouragement pour l'Industrie Nationale, à Paris. D'autres projections suivent, toujours devant un public trié sur le volet : le 11 juin pour le congrès des photographes à Lyon, le 11 juillet à la revue générale des sciences à Paris, le 10 novembre devant l'association belge de photographes à Bruxelles, le 16 novembre dans l'amphithéâtre de la Sorbonne, etc. Le cinématographe fait à chaque fois sensation. La première projection publique a lieu le 28 décembre 1895 au Salon Indien du Grand Café de l'hôtel Scribe, à Paris, devant trente-trois spectateurs. Le prix de la séance est fixé à 1 franc...

\section{acal $^{\text {bt }}$}

European leader in advanced technology solutions

\section{SOLUTIONS}

\section{POUR L'ECLAIRAGE}

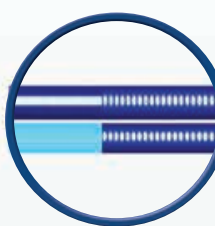

Diffuseurs de Lumière

Luminit

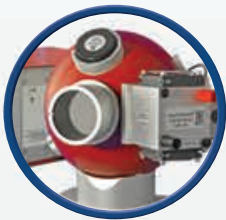

Sphères Intégrantes

(G) Gigahertz Optik

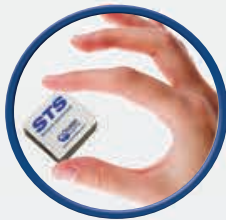

Spectromètres

cOcean

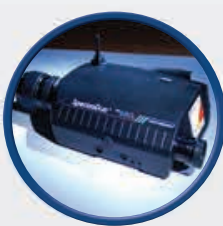

Spectrocolorimètres

TIPHOTO RESEARCH Hic

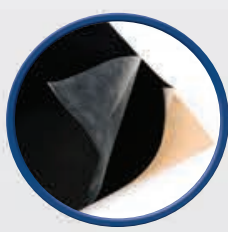

Pièges à Lumière

Acktar C

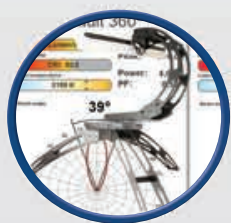

Goniophotomètres
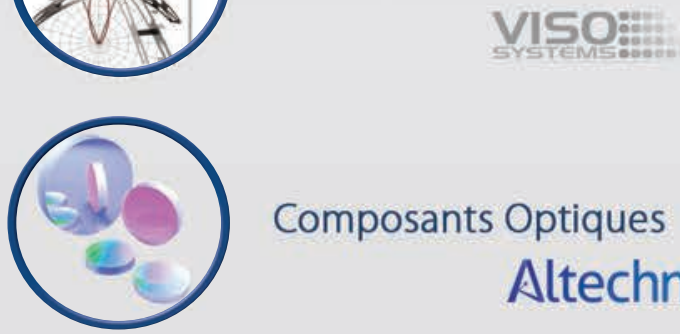

Composants Optiques

Altechna

WWw.acalbfi.fr

Tel +33(0)160795906 - photonique.fr@acalbfi.fr 
Le succès est immédiat. La première salle de cinéma est ouverte au $n^{\circ} 1$ de la rue de la République, à Lyon, le 25 janvier 1896. Moins de six mois plus tard, le cinématographe Lumière a franchi I'Atlantique et entamé la conquête du Nouveau-Monde : Minier et Pupier en font une démonstration à Montréal, au Canada! L'année 1895 marque aussi l'invention du concept de fiction cinématographique. Louis tourne, dans la foulée de son premier opus, le célèbre Arroseur Arrosé et fonde ce que le critique littéraire Ricciotto Canudo nomme en 1920 le septième art. Grâce à son expérience de photographe, Louis fait en effet preuve d'une maîtrise du cadrage et de la lumière qui distingue très vite ses réalisations de celles de ses prédécesseurs.

\section{Le succès industriel}

Notons qu'en commerciaux avisés, les frères Lumière donnent à leurs troupes, dès décembre 1895, l'ordre de fabriquer 200 cinématographes. Pourtant, il est de notoriété publique qu'ils considèrent leur appareil (leur moulin à images) comme « une invention sans avenir ». Ils laissent d'ailleurs à d'autres, notamment à Pathé ou Gaumont, le soin de faire du cinéma l'industrie florissante que nous connaissons. Dès 1907, la Société Lumière revient en effet à la seule production des produits photographiques, et les frères Lumière abandonnent définitivement l'exploitation du cinématographe. Ils se contentent désormais du rôle, peut-être plus modeste, de techniciens du cinéma. Et à ce titre, ils resteront longtemps à la pointe de l'innovation : citons, par exemple, l'invention en 1903 de la plaque photographique «autochrome », qui permet la couleur (on lui doit celle de nombreuses photos prises au cours de la Première Guerre Mondiale) ; l'invention en 1920 de la photostéréosynthèse qui permet la photographie en relief; ou encore en 1936 l'invention du cinéma en relief par le procédé des anaglyphes...
La mort d'Antoine en 1911 ne brise pas l'élan des frères Lumière. Ils font de leur industrie un empire prospère. Au milieu des années 1920, leurs usines couvrent $37000 \mathrm{~m}^{2}$ à Monplaisir, emploient 500 personnes et fabriquent des plaques et du papier photographiques, ainsi que des produits chimiques. Certaines de leurs inventions trouvent des applications en médecine, comme le tulle gras pour soigner les brûlés, ou l'allocaïne comme anesthésique dentaire... D'autres usines sont construites à Fontaines-sur-Saône, à Feyzin et aux États-Unis.

En 1946, Louis lègue à la cinémathèque française plus de 1800 vues qu'il a réalisées avec ses opérateurs. II meurt deux ans plus tard, le 6 juin 1948 à Bandol. Auguste lui survit 6 années, et s'éteint le 10 avril 1954. La ville de Lyon les classe parmi ses grands inventeurs et entrepreneurs, mais leur mémoire reste longtemps ternie par la sympathie qu'ils témoignèrent au régime fasciste de Mussolini et au gouvernement de Vichy pendant la Seconde Guerre. Ainsi, lorsqu'en 1995 la Banque de France veut honorer leur mémoire à l'occasion du centenaire de l'invention du cinéma, en imprimant un billet de 200 francs à leur effigie, elle se heurte à l'opposition virulente de l'Amicale des réseaux Action de la France Combattante, et les billets sont finalement détruits. Notons cependant qu'en 2012 la Monnaie de Paris parvient à éditer, sans souci notable, une pièce de 10 euros en argent représentant les frères Lumière, pour sa fameuse collection «Les Euros des Régions».

On le voit, dans la saga industrielle des Lumière, le cinéma n'aura finalement joué qu'un rôle modeste. En revanche, cette invention a apporté une renommée internationale, ainsi qu'une réputation tenace d'innovation, aux produits Lumière.

\section{Référence}

Les Lumière, B. Chardière, G. et M. Borgé (Bibliothèque des arts, Paris, 1985) Auguste et Louis Lumière, M. Faucheux (Gallimard, 2011)

\section{Venez célébrer « 2015, Année de la Lumière »}

\section{et découvrez tous nos ouvrages dans cette thématique !!}
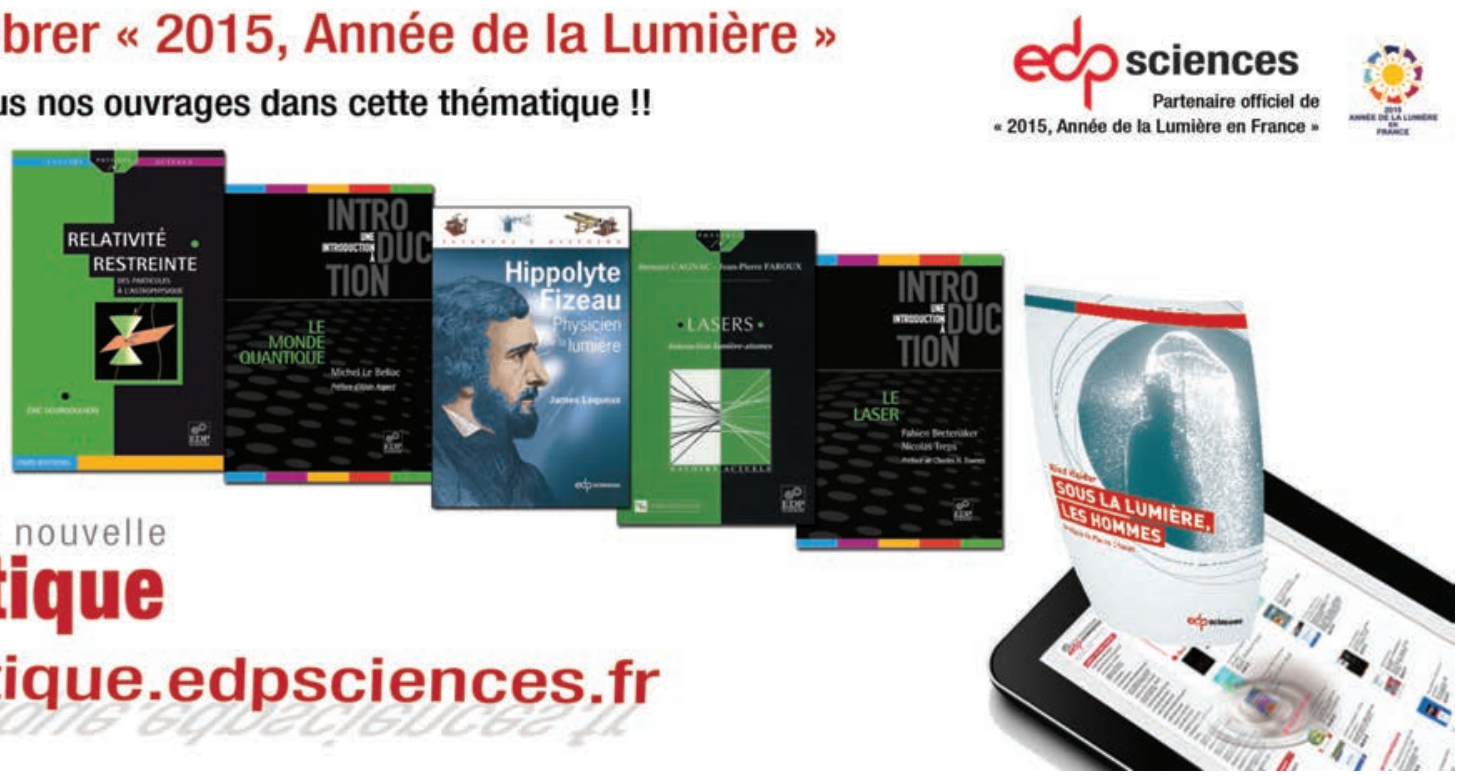